\title{
BMJ Open Comparison of three different exercise training modalities (aerobic, strength and mixed) in patients with schizophrenia: study protocol for a multicentre randomised wait-list controlled trial
}

\author{
Laura García-Garcés (D) , ${ }^{1}$ Sergio Lacamara Cano, ${ }^{2,3}$ Yago Cebolla Meliá, ${ }^{4}$ \\ María I Sánchez-López, ${ }^{1}$ David Marqués Azcona, ${ }^{5}$ Juan Francisco Lisón, 6,7 \\ Loreto Peyró-Gregori ${ }^{1}$
}

To cite: García-Garcés L, Lacamara Cano S, Cebolla Meliá Y, et al. Comparison of three different exercise training modalities (aerobic, strength and mixed) in patients with schizophrenia: study protocol for a multicentre randomised waitlist controlled trial. BMJ Open 2021;11:e046216. doi:10.1136/ bmjopen-2020-046216

- Prepublication history and additional supplemental material for this paper are available online. To view these files, please visit the journal online (http://dx.doi.org/10.1136/ bmjopen-2020-046216).

Received 30 October 2020 Accepted 24 August 2021

D) Check for updates

(c) Author(s) (or their employer(s)) 2021. Re-use permitted under CC BY-NC. No commercial re-use. See rights and permissions. Published by BMJ.

For numbered affiliations see end of article.

\section{Correspondence to}

Dr Laura García-Garcés;

lauragarciagarcesphd@gmail. com

\section{ABSTRACT}

Introduction Numerous studies support the practice of different physical exercise modalities as an effective treatment to address the problems associated with schizophrenia, reporting that they result in improvements in patient symptoms and quality of life. Given the lack of studies comparing different types of training in controlled environments, the aim of this proposed study will be to compare the effects of three physical exercise programmes (strength, aerobic and mixed) on the symptoms, body composition, level of physical activity and health-related quality of life of patients with schizophrenia. Methods and analysis A multicentre, single-blinded (evaluator), randomised, wait-list controlled (ratio 2:2:2:1) trial will be conducted with 105 patients recruited from different psychosocial care centres. The participants will be randomised into three 16-week training groups comprising 48 sessions lasting 1 hour each, or to the wait-list control group. The training groups will complete aerobic, strength or mixed (aerobic + strength) training. The participants will be assessed before, immediately after and 6 months after the end of the intervention. The patients in the wait-list control group $(n=15)$ will receive one of the three trainings immediately after the intervention. The study variables will include positive, negative and general symptomology (Positive and Negative Syndrome Scale) as the primary outcome; as secondary outcome: body composition (by assessing body mass index, body fat mass and waist circumference), physical activity levels (International Physical Activity Questionnaire-Short Form) and quality of life (abbreviated WHO Quality of Life questionnaire).

Ethics and dissemination This study was approved by the ethics committees for Biomedical Research at the CEU Cardenal Herrera University of Valencia, Spain (CEl18/215). Participants will be fully informed of the purpose and procedures of the study, and written informed consent will be obtained. The results from this study will be published in peer-reviewed journals and presented in scientific conferences.

Trial registration number NCT04987151.
Strengths and limitations of this study

- This is the first prospective randomised wait-list controlled trial to compare the effects of three different physical exercise programmes (aerobic, strength and mixed) in individuals with schizophrenia.

- This study assesses positive and negative psychotic symptoms, health-related quality of life and body composition.

- The statistical power is based on the primary objective to evaluate effects of physical exercise programmes on symptomatology.

- The nature of the physical exercise programmes (types of exercise, frequency, session duration, programme duration, intensity, progression and training settings) and the 6-month follow-up assessment are strengths of the study design.

- The study is limited by the absence of daily food records and for the lack of a control group for the analysis at 6 months.

\section{INTRODUCTION}

Schizophrenia is a serious chronic mental illness that, according to WHO data, ${ }^{1}$ affects 21 million people worldwide. This disease is characterised by a combination of positive symptoms (hallucinations, delusions, thoughts and/or movement disorders), negative symptoms (associability, anhedonia, abolition, affective flattening and alogia) and cognitive symptoms (problems with operational memory, executive functioning and concentration). ${ }^{23}$ In addition, schizophrenia is accompanied by a huge individual and social burden ${ }^{45}$ and is the eighth leading cause of disability-adjusted life-years in 15-44 years. ${ }^{6}$ 
Schizophrenia is related to a sedentary lifestyle ${ }^{7-9}$ and is associated with cardiovascular diseases, coronary heart disease,${ }^{10}$ diabetes, obesity, dyslipidaemia and metabolic syndrome, among other comorbidities. ${ }^{11}{ }^{12}$ Some of these pathologies are a consequence of the antipsychotic drugs that these patients receive to treat their disease, ${ }^{13}$ but there are also studies that postulate that the metabolic alterations present in these individuals are inherent to the schizophrenic disease they suffer. ${ }^{14}$ All of the above means that, compared with the general population, people suffering from this disease have a $40 \%-60 \%$ higher probability of premature death and a $20 \%$ lower life expectancy. ${ }^{15}$

On the other hand, there is evidence that the quality of life perceived by patients with schizophrenia is lower than in the rest of the population in every domain studied. ${ }^{16}$ The intensity of the symptoms of this disease, its treatment and the comorbidities associated with it strongly impact the quality of life of patients affected by it, which is further jeopardised by the social stigma and low selfesteem that it entails. ${ }^{17} 18$ Of note, some studies have shown that physical activity (PA) positively contributes to the quality of life of these patients. ${ }^{19}$

Without a doubt, PA is an important factor in preserving the general health and preventing chronic diseases such as diabetes, dyslipidaemia, obesity and cardiovascular diseases in individuals with schizophrenia. Indeed, in individuals with schizophrenia, exercise is inversely correlated with morbidity and mortality as a result of these diseases. ${ }^{20}$ Specifically, significant results in terms of quality of life, ${ }^{21}$ positive and negative symptoms, ${ }^{22-24}$ cognitive functioning ${ }^{25-28}$ improvement in sleep quality ${ }^{29}$ and cardiopulmonary function ${ }^{31-33}$ were found in studies that used PA as an intervention in populations affected by schizophrenia. In addition, PA reduces the general care burden of these patients. ${ }^{34}$

Therefore, the prescription of physical exercise is a practice validated for improving the symptoms of schizophrenia and to help prevent the diseases associated with it. However, to the best of our knowledge, there are still significant gaps in the evidence indicating what types of training might be most effective at improving the symptoms of these patients. ${ }^{21}{ }^{35-37}$ Most work studying the effects of PA in patients diagnosed with schizophrenia has focused on aerobic or mixed physical exercise programmes. ${ }^{12} 2330$ 32-34 38 In fact, even though strength training exercise interventions have shown improvements in diseases such as depression and anxiety, ${ }^{249}$ only two studies have used this type of training in patients with schizophrenia. ${ }^{40}$ Nonetheless, these studies found that strength training programmes reduced the psychopathology ${ }^{24}$ and improved the maximum strength and walking performance of these patients. ${ }^{2439}$

Based on all the above, the main objective of this proposed work will be to analyse and compare the effects of three different physical exercise programmes (strength, aerobic or mixed) on the symptomatology (positive and negative), health-related quality of life and anthropometric variables of patients with schizophrenia enrolled in a psychosocial rehabilitation programme.

\section{METHODS AND ANALYSIS}

\section{Study design}

This will be a four-armed, multicentre, single-blinded, randomised controlled trial (RCT), comparing four conditions: strength training, aerobic training, mixed training (strength +aerobic) and wait-list control group. The participants will be assessed at baseline, posttreatment and at a 6-month follow-up. All the patients in the wait-list control group will receive one of the three trainings immediately after the intervention, and will not be assessed at the 6-month follow-up. A flow chart showing the proposed progression of the participants through the study is shown in figure 1 . The work will adhere to the Consolidated Standards of Reporting Trials (CONSORT) standards for randomised trials ${ }^{41-43}$ as well as the CONSORT-Electronic and Mobile Health Applications and on Line Tele Health, ${ }^{44}$ the Standard Protocol Items: Recommendations for Intervention Trials guidelines (online supplemental additional file 1) and the WHO trial registration data set criteria (online supplemental additional file 2). ${ }^{45}$

\section{Patient involvement}

Patients will be involved at several stages of the trial, including the design, management and conduct of the trial. We will receive input from patients who are living with schizophrenia in the design of the trial materials and management oversight through membership of the trial steering committee. We carefully will assess the adverse events of the trial interventions on patients. We intend to disseminate the main results to trial participants and will seek patient and public involvement in the development of an appropriate method of dissemination.

\section{Study population, recruitment and eligibility criteria}

This RCT will be conducted from six psychosocial care centres for people with severe mental illness located in different parts of Spain: the Fundación Agustín Serrate (Huesca), Fundación Rey Ardid (Zaragoza), Fundación SASM (Valencia), Fundación Els Tres Turons (Barcelona), CREAP (Valencia) and Asociación ACOVA (Valencia). The participants will be recruited by the health staff working in the different centres.

The researchers who manage the study will go to the different psychosocial care centres to explain the study and eligibility criteria to the health staff, and will give them an information dossier containing the study characteristics and a detailed audiovisual manual with the description of each exercise intervention. The health staff at those institutions will then distribute the information to interested and suitable candidates directly via an interview in which the study will be explained in detail and they will be asked if they want to participate in the study. If they wish to participate, these patients will be 


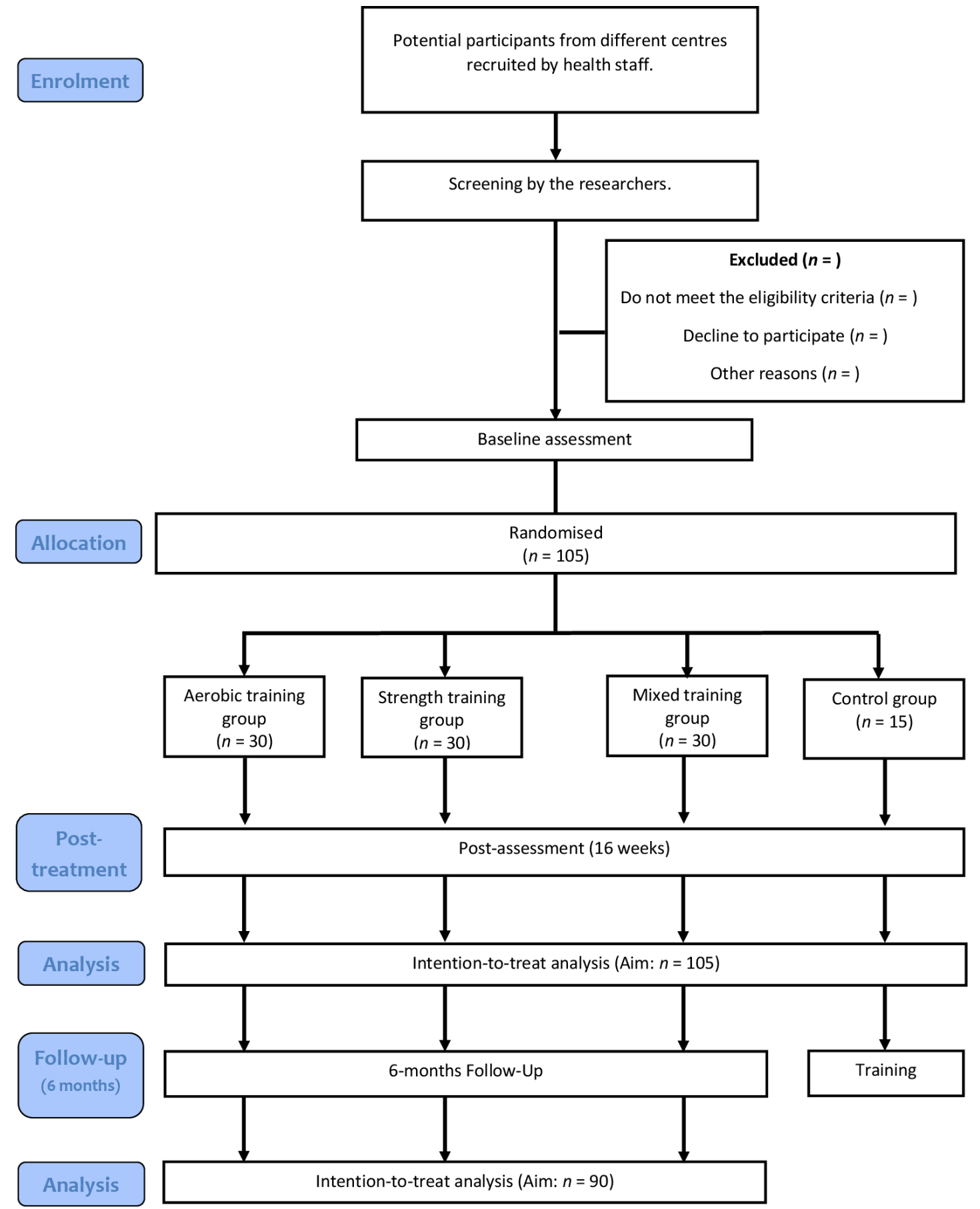

Figure 1 Flow chart representing the movement of the participants through the study.

asked to sign the informed consent document (online supplemental additional file 3 ) and will be instructed to maintain their usual treatments and appointments with mental health professionals.

To be included, the participants must fulfil all the inclusion criteria and none of the exclusion criteria. The inclusion criteria will be as follows: (1) age between 18 and 65 years; (2) Diagnostic and Statistical Manual of Mental Disorders, fifth Edition diagnosis of schizophrenia and (3) able to read and understand the Spanish language. The exclusion criteria will be: (1) acute suicidality; (2) representing an acute danger to others; (3) other psychiatric diagnoses or acute psychiatric illnesses; (4) other disorders that could prevent the person from completing the exercise training and (5) participation in similar programmes or interventions at the time of enrolment.

\section{Randomisation and blinding}

An independent researcher unaware of the study characteristics will perform the randomisation process. In order to randomly allocate the participants to one of the four conditions (aerobic, strength, mixed or wait-list control group), a computer-generated random number sequence $^{46}$ will be used (applying a simple allocation 
strategy). The randomisation will occur after baseline measures are taken and the allocation ratio (2:2:2:1) will be counterbalanced in each centre. This sequence will be recorded in a password-protected spreadsheet table and concealed to other researchers during the study.

Because the different exercise interventions significantly vary, it will be impossible to mask the group allocation to the physical therapists or the participants. However, the outcome evaluators and data analysts will be blinded to treatment allocations; outcome assessors and data analysts will be not involved in participant recruitment, treatment assignment and treatment administration (interventions). Participants will be instructed not to tell outcome assessors of the intervention they received. The success of blinding will be measured and reported using a blinding questionnaire. To avoid interobserver variability bias, the measurements in each of the groups will always be completed by the same investigator.

\section{Sample size}

Sample size calculation was conducted using G*Power software V.3.1.9. $2^{47}$ based on data collected from a similar study by Silva $e t a l^{24}$ The effect size $\left(\eta p^{2}\right)$ for the time by-group interaction in the positive symptoms of schizophrenia was 0.229 . To achieve $90 \%$ power, with an $\alpha$ level of 0.05 , the total sample size needed is 80 . Thus, anticipating a drop-out rate of $30 \%$ according to Vancampfort et $a l,{ }^{48}$ the necessary total sample size would be $(\mathrm{n}=105)$.

\section{Interventions}

The intervention will consist of a total of 48 sessions ( 3 weekly group-based sessions lasting 1 hour each for 16 weeks) and will be carried out at the gymnasium or the sports courts of each of the psychosocial care centres. To make the comparison fair, the total number of training sessions and duration of each session will be the same for the three training groups. These groups will be led by certified and experienced physical trainers (average experience of 5-10 years) from each psychosocial care centre who will also be responsible for recording each participant's degree of compliance with the intervention. The exercise dosing patters will be based on current recommendations for individuals with schizophrenia. ${ }^{49-51}$ The progression of the intensity of each training session will be a motivational strategy for the participants. To describe interventions, we have used the Consensus on Exercise Reporting Template (online supplemental additional file 4).

\section{Strength training}

Each strength training session will begin with a set of gentle stretching exercises lasting $10 \mathrm{~min}$, designed to target the major muscle groups. This will be followed by two sets of eight strength training exercises with 1 min of recovery programmed between each one. An elastic resistance band (Thera-band) will be used in four of the eight strength exercises. Finally, the training will end with 10 min of gentle stretching of the major muscle groups as a cool-down (figure 2).

The training intensity will increase over the 16 weeks of this intervention; the intensity of exercises completed without an elastic band will be amplified by increasing the number of repetitions the participants perform. For exercises performed with an elastic band, the intensity increase will be achieved by using the Borg Scale. ${ }^{52}$ This scale measures the effort an individual perceives when exercising and creates criteria to adjust the intensity of the programmed exercise.

In order to adequately use the Borg scale, the participants assigned to the strength training group must learn to use Theraband resistance bands on the first day and to easily identify, for each exercise, which gripping point on the band is equivalent to an effort that is moderate, intermediate, hard or very hard according to the Borg scale.

\section{Aerobic training}

Each session will begin with 10 min of stretching of the major muscle groups. Subsequently, participants will complete four series of brisk walking for $10 \mathrm{~min}$, followed by $1 \mathrm{~min}$ of recovery. To ensure that the intensity of the exercise progresses from moderate to vigorous, we will monitor the heart rate of each participant. The progression in exercise intensity will be achieved by increasing the participant's target HR every 2 weeks. Thus, using the formula published by Tanaka et al to calculate the maximum HR (MHR) (208-0.7 $\times$ age),${ }^{53}$ the intensity of the exercise will be progressively increased as follows: weeks 1-2: 55\% MHR; weeks 3-4: 58\% MHR; weeks 5-6: 61\% MHR; weeks 7-8: 64\% MHR; weeks 9-10: 67\% MHR; weeks 11-12: 70\% MHR; weeks 13-14: 73\% MHR; and weeks 15-16: 76\% MHR. The session will end with a 10 min session of gentle stretching exercises targeting the major muscle groups (figure 3 ).

\section{Mixed training}

As in the previous two groups, each training session will begin with $10 \mathrm{~min}$ of stretching of the major muscle groups. The main part of each mixed session will consist of two parts. First, similar to the strength training group, the participants will perform a single circuit of eight strength exercises interspersed with 1 min of recovery for each strength exercise. Second, as in the aerobic training group, the participants will perform two sets brisk walking for $10 \mathrm{~min}$, followed by $1 \mathrm{~min}$ of recovery, following the same exercise intensity progression as described for the aerobic training group. Finally, these sessions will also end with a 10 min session of gentle stretching exercises targeting the major muscle groups.

\section{Instruments}

The participants will be assessed at three different times. First, before beginning the intervention; second, immediately after the end of the intervention; and third, 6 months after the end of the intervention (6-month follow-up). All the assessments will be performed in one single session 

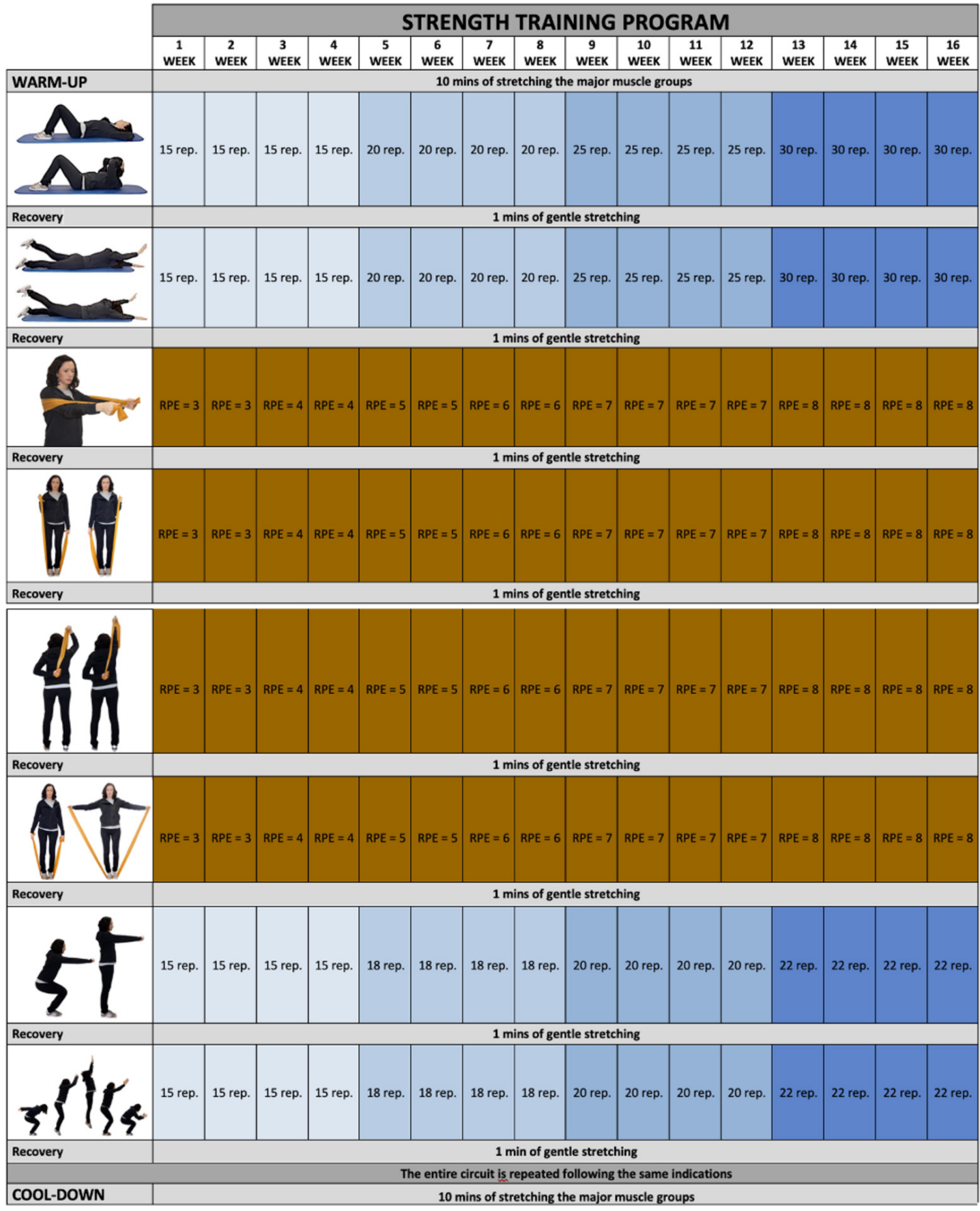

Figure 2 Strength training. Borg Rating of Perceived Exertion (RPE). rep, repetition.

and will be scheduled between 10:00 and 12:00 hours to minimise variability.

Variables and evaluation times are summarised in table 1.

\section{Metrics}

The psychometric attributes of all the measurement tools used in this project, such as the reliability and validity, are psychometrically sound.

\section{Primary outcome}

The Positive and Negative Syndrome Scale (PANSS) is a semistructured interview which assesses the positive
(PANSS-P: 7 items, range 7-49), negative (PANSS-N: 7 items, range 7-49) and general (PANSS-G: 16 items, range 16-112) symptoms of psychosis experienced by patients in the week prior to the test on a 7-point Likerttype scale (from 1, 'none', to 7 , 'extreme'). ${ }^{54}$ We will analyse the three subscales separately and the positivesymptom factor will serve as the primary outcome of this study. The subscales of the Spanish version are strongly associated with those of the original version ( $\mathrm{r}=0.92$ for PANSS-P and $r=0.83$ for PANSS-N), with item correlations ranging from $r=0.64$ to $r=0.97$, and with high inter-rater reliability $(\mathrm{r}=0.81) .{ }^{55}$ 


\begin{tabular}{|c|c|c|c|c|c|c|c|c|c|c|c|c|c|c|c|c|}
\hline \multirow[b]{2}{*}{ PROGRAM } & \multicolumn{16}{|c|}{ AEROBIC TRAINING PROGRAM } \\
\hline & $\begin{array}{c}1 \\
\text { WEEK }\end{array}$ & $\begin{array}{c}2 \\
\text { WEEK }\end{array}$ & $\begin{array}{c}3 \\
\text { WEEK }\end{array}$ & $\begin{array}{c}4 \\
\text { WEEK }\end{array}$ & $\begin{array}{c}5 \\
\text { WEEK }\end{array}$ & $\begin{array}{c}6 \\
\text { WEEK }\end{array}$ & $\begin{array}{c}7 \\
\text { WEEK }\end{array}$ & $\begin{array}{c}8 \\
\text { WEEK }\end{array}$ & $\begin{array}{c}9 \\
\text { WEEK }\end{array}$ & $\begin{array}{c}10 \\
\text { WEEK }\end{array}$ & $\begin{array}{c}11 \\
\text { WEEK }\end{array}$ & $\begin{array}{c}12 \\
\text { WEEK }\end{array}$ & $\begin{array}{c}13 \\
\text { WEEK }\end{array}$ & $\begin{array}{c}14 \\
\text { WEEK }\end{array}$ & $\begin{array}{c}15 \\
\text { WEEK }\end{array}$ & $\begin{array}{c}16 \\
\text { WEEK }\end{array}$ \\
\hline WARM-UP & \multicolumn{16}{|c|}{10 mins of stretching the major muscle groups } \\
\hline AEROBIC TRAINING & \multicolumn{16}{|c|}{$\begin{array}{l}10 \text { minutes of brisk walking. The exercise intensity should be moderate. We will control it by monitoring the heart rate (HR). Any patients who cannot } \\
\text { use their HR as a reference because of their medical treatments should perform the exercise at an intensity which makes it difficult but not impossible } \\
\text { for them to speak while performing it. }\end{array}$} \\
\hline HR MEASUREMENT & \multicolumn{16}{|c|}{ See the proposed HR table created based on the patient age and the training program exercise week. } \\
\hline AEROBIC TRAINING & \multicolumn{16}{|c|}{$\begin{array}{l}\mathbf{1 0} \text { minutes of brisk walking. The exercise intensity should be moderate. We will control it by monitoring the heart rate (HR). Any patients who cannot } \\
\text { use their HR as a reference because of their medical treatments should perform the exercise at an intensity which makes it difficult but not impossible } \\
\text { for them to speak while performing it. }\end{array}$} \\
\hline HR MEASUREMENT & \multicolumn{16}{|c|}{ See the proposed HR table created based on the patient age and the training program exercise week. } \\
\hline AEROBIC TRAINING & \multicolumn{16}{|c|}{$\begin{array}{l}10 \text { minutes of brisk walking. The exercise intensity should be moderate. We will control it by monitoring the heart rate (HR). Any patients who cannot } \\
\text { use their HR as a reference because of their medical treatments should perform the exercise at an intensity which makes it difficult but not impossible } \\
\text { for them to speak while performing it. }\end{array}$} \\
\hline HR MEASUREMENT & \multicolumn{16}{|c|}{ See the proposed HR table created based on the patient age and the training program exercise week. } \\
\hline AEROBIC TRAINING & \multicolumn{16}{|c|}{$\begin{array}{l}10 \text { minutes of brisk walking. The exercise intensity should be moderate. We will control it by monitoring the heart rate (HR). Any patients who cannot } \\
\text { use their HR as a reference because of their medical treatments should perform the exercise at an intensity which makes it difficult but not impossible } \\
\text { for them to speak while performing it. }\end{array}$} \\
\hline HR MEASUREMENT & \multicolumn{16}{|c|}{ See the proposed HR table created based on the patient age and the training program exercise week. } \\
\hline COOL-DOWN & \multicolumn{16}{|c|}{ At the end of the second set: 10 mins of stretching the major muscle groups } \\
\hline
\end{tabular}

Figure 3 Aerobic training.

\section{Secondary outcomes}

Anthropometric and body composition variables: The body mass index, calculated as the patient weight in kilograms divided by their height in squared metres, will be calculated using a SECA 780 electronic balance scale with a mechanical telescopic stadiometer. Body fat mass will be determined using a TANITA TBF-410 M body-fat analyser. Waist circumference will be measured to the nearest centimetre using a flexible tape measure at the level half-way between the lower rib margin and the iliac crest.
PA levels: PA levels will be assessed using the International Physical Activity Questionnaire-Short Form (IPAQ$\mathrm{SF}) .{ }^{56}$ Using seven items, this self-reported questionnaire collects data on the patients' PA in the 7 days prior to the test. The total number of days and minutes of PA will be calculated by adding all PA category scores performed over the 7 days. Specifically, the IPAQ-SF questionnaire records activity at four intensity levels: (1) vigorous activity such as aerobics; (2) moderate activity such as leisure cycling; (3) walking and (4) sitting. This makes

Table 1 Study variables and assessment points

\begin{tabular}{|c|c|c|c|c|c|}
\hline \multirow[b]{3}{*}{ Time point } & \multicolumn{5}{|c|}{ Study period } \\
\hline & \multicolumn{2}{|c|}{ Enrolment } & \multirow{2}{*}{$\begin{array}{l}\text { Allocation } \\
0 \\
\end{array}$} & \multirow{2}{*}{$\begin{array}{l}\text { Postallocation } \\
t_{2} \\
\text { Post-treatment }\end{array}$} & \multirow{2}{*}{$\begin{array}{l}\text { Close-out } \\
t_{3} \\
\text { 6-month follow-up }\end{array}$} \\
\hline & $-t_{1}$ & $\begin{array}{l}t_{1} \\
\text { baseline }\end{array}$ & & & \\
\hline \multicolumn{6}{|l|}{ Enrolment } \\
\hline Eligibility screen & $\mathrm{x}$ & & & & \\
\hline Informed consent & $\mathrm{x}$ & & & & \\
\hline Allocation & & & $\mathrm{x}$ & & \\
\hline \multicolumn{6}{|l|}{ Interventions } \\
\hline \multicolumn{6}{|l|}{ (Strength training) } \\
\hline \multicolumn{6}{|l|}{ (Aerobic training) } \\
\hline \multicolumn{6}{|l|}{ (Mixed training) } \\
\hline \multicolumn{6}{|l|}{ (Control group) } \\
\hline \multicolumn{6}{|l|}{ Assessments } \\
\hline Positive psychotic symptoms & & $\mathrm{x}$ & & $\mathrm{x}$ & $\mathrm{x}$ \\
\hline Negative psychotic symptoms & & $\mathrm{x}$ & & $\mathrm{x}$ & $\mathrm{x}$ \\
\hline General psychopathology & & $\mathrm{x}$ & & $\mathrm{x}$ & $\mathrm{x}$ \\
\hline Body mass index & & $\mathrm{x}$ & & $\mathrm{x}$ & $\mathrm{x}$ \\
\hline Body fat mass & & $\mathrm{x}$ & & $\mathrm{x}$ & $x$ \\
\hline Waist circumference & & $\mathrm{x}$ & & $\mathrm{x}$ & $\mathrm{x}$ \\
\hline Quality of life & & $\mathrm{x}$ & & $x$ & $x$ \\
\hline Level of physical activity & & $\mathrm{x}$ & & $\mathrm{x}$ & $\mathrm{x}$ \\
\hline
\end{tabular}


it possible to classify the PA levels of the participants as 'high', 'moderate or 'low' ${ }^{57}$ The IPAQ has been validated in 12 countries $^{58}$ and showed adequate psychometric properties and the short version (the IPAQ-SF) has shown acceptable validity in an adult Spanish population. ${ }^{59}$

The abbreviated WHO Quality of Life AssessmentBREF: ${ }^{60}$ This survey comprises 26 items with five Likerttype responses each, and is a standard questionnaire used to measure patient quality of life. It assesses patients under four health domains: physical, psychological, social and environmental. In this study, we will analyse the sum of the four dimensions, with higher scores indicating a better quality of life. This scale has been validated for Spanish and the instrument has a good internal consistency with a Cronbach alpha of 0.88 for the overall scale and a range of $0.70-0.79$ for its dimensions. ${ }^{61}$

\section{Sociodemographic metrics}

Age, gender, marital status, education level, job status and institutionalisation regime will be encoded.

\section{Clinical metrics}

The duration of patient psychoses and history of hospitalisations since the first episode will be recorded. Other pharmacological and non-pharmacological interventions, as well as current medication and psychosocial care, will also be checked. Adverse events to the interventions will be also registered.

\section{Adherence}

Specialists will direct all 48 sessions in each of the three training groups, registering each participant's attendance for each session, and adverse or unintended effects. Specialist will promote participant retention and complete follow-up. Sessions will be marked as finished when at least $75 \%$ of the training was completed. Participants will be instructed not to perform other rehabilitation interventions programmes outside of the intervention for the entire duration of the study.

\section{Statistical data analysis}

Based on an intention-to-treat sample, two-way mixed analysis of covariance (ANCOVA) $(2 \times 4)$ tests will be used to compare how the study interventions affect the primary and secondary outcomes, using time (baseline and postintervention-primary end point-) as the within-group factor and group (aerobic, strength, mixed or wait-list control) as the between-group factor. Two-way mixed ANCOVA $(3 \times 3)$ tests will also be used to compare how the study interventions affect the outcomes, using time (baseline, postintervention and 6-month follow-up) as the within-group factor and group (aerobic, strength or mixed) as the between-group factor. The analysis will be adjusted for sex, age, adherence and antipsychotic medications. Effect sizes will be estimated using the partial eta squared formula $(\eta 2 p)$ and interpreted following the Cohen guidelines ${ }^{62}$ for small effect sizes $(\eta 2 \mathrm{p}=0.01)$, moderate effect sizes $(\eta 2 \mathrm{p}=0.06)$, and large effect sizes $(\eta 2 p=0.14) \cdot \chi^{2}$ will be used to statistically assess success of blinding. The significance level will be set at $5 \%$ (twotailed analyses) and the data will be analysed using SPSS software, V.24.0 (IBM).

\section{Data monitoring}

The data monitoring committee will comprise at least two independent members that will periodically check the progression of the trial in the six psychosocial care centres. After randomising the participants, the committee will meet every 6 weeks to review a report submitted by the researchers for the purpose of monitoring the progress of recruitment and data collection. The data monitoring committee will do an interim analysis immediately after the end of the intervention, in order to decide to finish the trial. If any important modifications are made to the protocol, these will be communicated to the Ethics Committee at once.

\section{Data confidentiality}

After the measurements are recorded, the collected data will be transferred to a database on a password-locked stand-alone desktop computer which will be kept in a locked research room at the Department of Medicine in the Faculty of Health Sciences at the University CEUCardenal Herrera of Valencia. The collected data will be saved as traceable anonymous data with sequentially allocated numbers which the researchers will be able to access.

\section{ETHICS AND DISSEMINATION}

This study will be conducted according to the principles established in the Declaration of Helsinki, the Convention on Human Rights and Biomedicine (Oviedo Convention), and the UNESCO Universal Declaration on the human genome research and human rights. This project was approved by the Ethics Committee for Biomedical Research at the CEU Cardenal Herrera University of Valencia in Spain (reference number: CEI18/215) (online supplemental additional file 5); the ethics approval applies to all participating centres.

All the participants will be informed about the length and characteristics of the study and the voluntary nature of their participation in it. After explaining the project in detail, we will answer any questions potential participants might have about it and then they will be provided with an informed consent document that they will have to sign should they wish to participate in the study. In turn, we will provide them with the contact details for the principal investigator of the project so participants will be able to communicate with them at any time.

Participants will also be informed that all the data collected during the investigation will be treated confidentially in accordance with current regulations on the protection of personal data, Organic Law 3/2018, of December 5 , on the protection of personal data and guarantee of digital rights, and European Union regulation 2016/679 of the European Parliament and Council, of 27 
April 2016, regarding the protection of natural persons with regard to the processing of personal data and the free circulation of this data.

The findings of this study will be published in peerreviewed indexed (Journal Citation Reports) journals. We will also present the results and findings at related research conferences. Furthermore, we will also make the full study report available to the relevant health authorities.

\section{DISCUSSION}

The greatest strength of this study is that, to the best of our knowledge, it will be the first RCT to compare the effects of three types of training programme (aerobic, strength or mixed) on improving the symptoms of psychosis.

Many studies have been published that demonstrate the benefits that performing physical exercise has on the population affected by schizophrenia, ${ }^{12} 1333963$ and therefore, this type of non-pharmacological therapeutic strategy should be one of the standard treatments prescribed to these patients. Some studies have examined the benefits of aerobic training, ${ }^{13} 2363$ others have focused on mixed training interventions, ${ }^{1238}$ and still others have compared these strategies or implemented more sedentary activities such as occupational therapy. ${ }^{33}$ Some work has also evaluated the effects of practising yoga, ${ }^{23}$ dance ${ }^{64}$ or football. ${ }^{22}$ However, only two studies have evaluated the effectiveness of strength training in patients with schizophrenia. ${ }^{24} 39$

The work by Heggelund $e t a \vec{l}^{9}$ evaluated the effects that training the maximum lower-limb strength for 8 weeks had on the net mechanical efficiency of walking, the symptoms of schizophrenia and patient quality of life, and compared these outcomes with the effects of a sedentary activity such as self-entertainment with video games. Their results suggested that this type of strength training improved the maximum lower-limb strength of these patients as well as their walking performance, however, they found no alterations in the overall PANSS or 36-Item Short Form Health Survey scores.

In contrast, the study by Silva $e t a l^{24}$ assessed the differences between the effects of 20 weeks of strength training vs mixed training on the symptoms of psychosis or depression, quality of life and serum concentrations of insuline growth factor-1, insuline growth factor binding protein and a neurotrophic factor derived from brain brain-derived neurotrophic factor in patients with schizophrenia. This group found statistically significant improvements for both the strength and the mixed training groups in the overall PANSS scale score, positive symptomatology and maximum strength in the arm-extension test. Statistically significant improvements in the negative symptomatology and maximum strength in the chest-press test were only found in the strength training group. Although the results of these two publications are encouraging, further investigation will be required because the sample size in both these studies was small, with a maximum of only 13 participants per group, and neither of them collected data from a follow-up phase.
In addition, one of these studies did not use a randomised sampling strategy. ${ }^{39}$

Strength training has also obtained good results in other lines of research enquiry. For example, Cassilhas et $a b^{66}$ concluded that intensive strength training conducted in an elderly population improved their mood, anxiety and strength. Similarly, Stanton et al reviewed the benefits of aerobic and strength training in patients with depression and found that the latter was able to improve the mood and symptoms of depression in these patients. ${ }^{67}$ However, these results strongly differ from those from a meta-analysis carried out by Gordon et al which concluded that strength training significantly reduced the symptoms of depression. ${ }^{40}$

A cross-sectional study concluded that patients with schizophrenia showed lower hand grip strength scores compared with healthy controls, and that hand grip strength scores correlated positively with cognitive functions. ${ }^{68} \mathrm{~A}$ more recent study concluded that higher hand grip strength was associated with greater left and right hippocampal volume and reduced white matter hyperintensities in major depressive disorder (MDD). These authors considered that interventions targeting strength fitness could improve brain health and reduce the neurocognitive abnormalities associated with MDD. ${ }^{69}$ Finally, Subramaniapillai et $a l^{70}$ conducted a descriptive study with 113 patients diagnosed with schizophrenia and 60 patients with bipolar disorder to determine their PA preferences, and $67.6 \%$ of the respondents subsequently stated that they would like incorporate strength training into their exercise programmes.

While the mechanisms by which the different exercise interventions may influence the symptoms and cognition of our patients will extend beyond the scope of this study, several mechanisms have been proposed in the scientific literature. The most frequently cited are neuroprotective mechanisms such as decreased inflammation, increased neurogenesis and neuroplasticity via brain-derived neurotrophic factor, and remyelination of white matter tracts. ${ }^{71}$

Considering all the above, and given that so far no studies have identified which training types are most beneficial to patients affected by schizophrenia, the study plan described here aims to analyse and compare the effects of strength training, aerobic training and mixed training interventions on the symptomatology, health-related quality of life and anthropometric variables of these patients. The design of this wait-list controlled study incorporates a series of improvements with respect to previously published work examining the effects of strength training in patients with schizophrenia: this will be a multicentre study, adequately powered $(n=105)$, and a follow-up assessment carried out 6 months after the end of the intervention. Finally, we will be able to compare the benefits of each of the main types of training because we will include three intervention groups, and we will report of all the exercise training programmes information (types of exercise, frequency, session duration, programme duration, intensity, progression, training settings (ie, supervised or group sessions)).

Nevertheless, this study will have some limitations. First, we do not plan to record the dietary intake of the participants 
and so it will be impossible to independently assess the impact of physical exercise on anthropometric parameters and body composition. Second, the questionnaire data (level of PA and quality of life) will be self-reported, which may be affected by participants' personal perceptions. Third, it should be noted-when interpreting the results-that the combined exercise type group (aerobic +strength) will not include a full dose of either of the treatments alone. Finally, the lack of a control group for the analysis at 6 months should be considered when interpreting the results at this point.

The results of this project will allow us to separately understand the effects of each of the training interventions and identify if any of them are more beneficial to these patients with schizophrenia in terms of the different variables we plan to analyse. This knowledge will help to improve the prescription of different training types to each patient to help them maintain good control of symptoms of the disease.

\section{TRIAL STATUS}

Protocol version date: 26 July 2021

Date recruitment began: October 2021

Approximate date when recruitment will be completed: January 2022

\section{Author affiliations \\ ${ }^{1}$ Department of Nursing and Physiotherapy, Faculty of Health Sciences, Universidad Cardenal Herrera-CEU,CEU Universities, Moncada, Valencia, Spain ${ }^{2}$ State Reference Center for Psychosocial Rehabilitation (CREAP), Valencia, Spain ${ }^{3}$ Department of Sociology of Public Policies of the University of Zaragoza, Zaragoza, Spain \\ ${ }^{4}$ Department of Universal Health and Public Health, Valencia, Spain \\ ${ }^{5}$ Vice Presidency and Ministry of Equality and Inclusive Policies, Valencia, Spain \\ ${ }^{6}$ Department of Biomedical Science, Faculty of Health Sciences, Universidad Cardenal Herrera-CEU,CEU Universities, Moncada, Valencia, Spain \\ ${ }^{7}$ Centre of Networked Biomedical Research in the Physiopathology of Obesity and Nutrition (CIBERobn), CB06/03, Carlos III Health Institute, Madrid, Spain}

Correction notice This article has been corrected since it was published. Affiliation of Sergio Lacamara Cano has been updated.

Twitter María I Sánchez-López @I have not twitter

Acknowledgements We would like to thank care centres: Fundación Agustín Serrate, Fundación Rey Ardid, Fundación SASM, Fundación Els Tres Turons, CREAP, and Asociación ACOVA. This work was supported by the Generalitat Valenciana (AIC0/2019/331) and by the University CEU Cardenal Herrera (ICLINIC19/02). CIBERobn is an initiative of ISCIII.

Contributors LG-G: Conceived the study and wrote the draft for the manuscript. LG-G, SLC, YCM, MIS-L, JFL, DMA and LP-G contributed to the development of the design. SLC and LP-G contributed to the literature search. All authors contributed to refinement of the study protocol and approved the final manuscript.

Funding The authors have not declared a specific grant for this research from any funding agency in the public, commercial or not-for-profit sectors.

Competing interests None declared.

Patient consent for publication Not required.

Provenance and peer review Not commissioned; externally peer reviewed.

Supplemental material This content has been supplied by the author(s). It has not been vetted by BMJ Publishing Group Limited (BMJ) and may not have been peer-reviewed. Any opinions or recommendations discussed are solely those of the author(s) and are not endorsed by BMJ. BMJ disclaims all liability and responsibility arising from any reliance placed on the content. Where the content includes any translated material, BMJ does not warrant the accuracy and reliability of the translations (including but not limited to local regulations, clinical guidelines, terminology, drug names and drug dosages), and is not responsible for any error and/or omissions arising from translation and adaptation or otherwise.

Open access This is an open access article distributed in accordance with the Creative Commons Attribution Non Commercial (CC BY-NC 4.0) license, which permits others to distribute, remix, adapt, build upon this work non-commercially, and license their derivative works on different terms, provided the original work is properly cited, appropriate credit is given, any changes made indicated, and the use is non-commercial. See: http://creativecommons.org/licenses/by-nc/4.0/.

ORCID iD

Laura García-Garcés http://orcid.org/0000-0003-4103-3960

\section{REFERENCES}

1 World Health Organization. Schizophrenia, 2019. Available: https:// www.who.int/news-room/fact-sheets/detail/schizophrenia [Accessed 07 Jan 2020].

2 van der Gaag M, Hoffman T, Remijsen M, et al. The five-factor model of the positive and negative syndrome scale II: a ten-fold crossvalidation of a revised model. Schizophr Res 2006;85:280-7.

3 Heinrichs RW, Zakzanis KK. Neurocognitive deficit in schizophrenia: a quantitative review of the evidence. Neuropsychology 1998;12:426-45.

4 Knapp M, Mangalore R, Simon J. The global costs of schizophrenia. Schizophr Bull 2004;30:279-93.

5 Whiteford HA, Degenhardt L, Rehm J, et al. Global burden of disease attributable to mental and substance use disorders: findings from the global burden of disease study 2010. Lancet 2013;382:1575-86.

6 World Health Organization. International classification of functioning, disability and health (ICF). Geneva: World Health Organization, 2001.

7 Faulkner G, Cohn T, Remington G. Validation of a physical activity assessment tool for individuals with schizophrenia. Schizophr Res 2006;82:225-31.

8 Gorczynski P, Faulkner G. Exercise therapy for schizophrenia. Cochrane Database Syst Rev 2010;5:CD004412.

9 McLeod HJ, Jaques S, Deane FP. Base rates of physical activity in Australians with schizophrenia. Psychiatr Rehabil J 2009;32:269-75.

10 Hennekens $\mathrm{CH}$, Hennekens AR, Hollar D, et al. Schizophrenia and increased risks of cardiovascular disease. Am Heart $J$ 2005;150:1115-21.

11 Ainsworth BE, Haskell WL, Herrmann SD, et al. 2011 compendium of physical activities: a second update of codes and Met values. Med Sci Sports Exerc 2011;43:1575-81.

12 Marzolini S, Jensen B, Melville P. Feasibility and effects of a groupbased resistance and aerobic exercise program for individuals with severe schizophrenia: a multidisciplinary approach. Ment Health Phys Act 2009;2:29-36.

13 Beebe LH, Tian L, Morris N, et al. Effects of exercise on mental and physical health parameters of persons with schizophrenia. Issues Ment Health Nurs 2005;26:661-76.

14 Orellana G, Rodríguez M, González N, et al. Esquizofrenia Y SU asociación Con Enfermedades médicas crónicas. Rev. méd. Chile 2017; 145:1047-53.

15 World Health Organization. Mental health action plan 2013-2020, 2013. Available: https://apps.who.int/iris/bitstream/handle/10665/ 89966/9789241506021_eng.pdf?sequence=1 [Accessed 07 Jan 2020].

16 Dompablo M. Calidad de vida en esquizofrenia [PhD]. Spain: Facultad de Medicina, Universidad Complutense de Madrid, 2018.

17 Hjorth $\mathrm{P}$, Medici CR, Juel A, et al. Improving quality of life and physical health in patients with schizophrenia: a 30-month program carried out in a real-life setting. Int J Soc Psychiatry 2017;63:287-96.

18 Palijan Tija Žarković, Kovačević D, Vlastelica M, et al. Quality of life of persons suffering from schizophrenia, psoriasis and physical disabilities. Psychiatr Danub 2017;29:60-5.

19 Vancampfort D, Guelinckx H, Probst M, et al. Health-Related quality of life and aerobic fitness in people with schizophrenia. Int J Ment Health Nurs 2015;24:394-402.

20 Harrington M, Gibson S, Cottrell RC. A review and meta-analysis of the effect of weight loss on all-cause mortality risk. Nutr Res Rev 2009;22:93-108.

21 Poulin J, Daoust A-M, Forest G, et al. Sleep architecture and its clinical correlates in first episode and neuroleptic-naive patients with schizophrenia. Schizophr Res 2003;62:147-53.

22 Battaglia G, Alesi M, Inguglia M, et al. Soccer practice as an add-on treatment in the management of individuals with a diagnosis of schizophrenia. Neuropsychiatr Dis Treat 2013;9:595-603. 
23 Duraiswamy G, Thirthalli J, Nagendra HR, et al. Yoga therapy as an add-on treatment in the management of patients with schizophrenia--a randomized controlled trial. Acta Psychiatr Scand 2007;116:226-32.

24 Silva BAE, Cassilhas RC, Attux C, et al. A 20-week program of resistance or concurrent exercise improves symptoms of schizophrenia: results of a blind, randomized controlled trial. Braz J Psychiatry 2015;37:271-9.

25 Firth J, Stubbs B, Rosenbaum S, et al. Aerobic exercise improves cognitive functioning in people with schizophrenia: a systematic review and meta-analysis. Schizophr Bull 2017;43:546-56.

26 Choi J, Taylor B, Fiszdon JM, et al. The synergistic benefits of physical and cognitive exercise in schizophrenia: promoting motivation to enhance community effectiveness. Schizophr Res Cogn 2020;19:100147.

27 Maurus I, Röh A, Falkai P, et al. Nonpharmacological treatment of dyscognition in schizophrenia: effects of aerobic exercise. Dialogues Clin Neurosci 2019;21:261-9.

28 van der Stouwe ECD, van Busschbach JT, de Vries B, et al. Neural correlates of exercise training in individuals with schizophrenia and in healthy individuals: a systematic review. Neuroimage Clin 2018;19:287-301.

29 Lalande D, Thériault L, Kalinova Émilia, et al. The effect of exercise on sleep quality and psychological, physiological, and biological correlates in patients with schizophrenia: a pilot study. Schizophr Res 2016;171:235-6.

30 Leone M, Lalande D, Thériault L, et al. Effects of an exercise program on the physiological, biological and psychological profiles in patients with mood disorders: a pilot study. Int $J$ Psychiatry Clin Pract 2018;22:268-73.

31 Armstrong HF, Bartels MN, Paslavski O, et al. The impact of aerobic exercise training on cardiopulmonary functioning in individuals with schizophrenia. Schizophr Res 2016;173:116-7.

32 Scheewe TW, Takken T, Kahn RS, et al. Effects of exercise therapy on cardiorespiratory fitness in patients with schizophrenia. Med Sci Sports Exerc 2012;44:1834-42.

33 Scheewe TW, van Haren NEM, Sarkisyan G, et al. Exercise therapy, cardiorespiratory fitness and their effect on brain volumes: a randomised controlled trial in patients with schizophrenia and healthy controls. Eur Neuropsychopharmacol 2013;23:675-85.

34 Scheewe TW, Backx FJG, Takken T, et al. Exercise therapy improves mental and physical health in schizophrenia: a randomised controlled trial. Acta Psychiatr Scand 2013;127:464-73.

35 Czobor P, Volavka J, Sheitman B, et al. Antipsychotic-Induced weight gain and therapeutic response: a differential association. J Clin Psychopharmacol 2002;22:244-51.

36 Dauwan M, Begemann MJH, Heringa SM, et al. Exercise improves clinical symptoms, quality of life, global functioning, and depression in schizophrenia: a systematic review and meta-analysis. Schizophr Bull 2016;42:588-99.

37 Keller-Varady K, Varady PA, Röh A, et al. A systematic review of trials investigating strength training in schizophrenia spectrum disorders. Schizophr Res 2018;192:64-8.

$38 \mathrm{Kim} \mathrm{H}$-jae, Song B-kil, So B, et al. Increase of circulating BDNF levels and its relation to improvement of physical fitness following 12 weeks of combined exercise in chronic patients with schizophrenia: a pilot study. Psychiatry Res 2014;220:792-6.

39 Heggelund J, Morken G, Helgerud J, et al. Therapeutic effects of maximal strength training on walking efficiency in patients with schizophrenia - a pilot study. BMC Res Notes 2012;5:344.

40 Gordon BR, McDowell CP, Hallgren M, et al. Association of efficacy of resistance exercise training with depressive symptoms. JAMA Psychiatry 2018;75:566-76.

41 Schulz KF, Altman DG, Moher D, et al. Consort 2010 statement: updated guidelines for reporting parallel group randomised trials. BMJ 2010;340:c332

42 Moher D, Hopewell S, Schulz KF, et al. Consort 2010 explanation and elaboration: updated guidelines for reporting parallel group randomised trials. J Clin Epidemiol 2010;63:e1-37.

43 Moher D, Schulz KF, Altman DG. The CONSORT statement: revised recommendations for improving the quality of reports of parallelgroup randomised trials. Lancet 2001;357:1191-4.

44 Eysenbach G, CONSORT-EHEALTH Group. CONSORT-EHEALTH: improving and standardizing evaluation reports of web-based and mobile health interventions. J Med Internet Res 2011;13:e126.

45 Chan A-W, Tetzlaff JM, Gøtzsche PC, et al. Spirit 2013 explanation and elaboration: guidance for protocols of clinical trials. BMJ 2013;346:e7586.
46 Saghaei M. Random allocation software for parallel group randomized trials. BMC Med Res Methodol 2004;4:26.

47 Faul F, Erdfelder E, Lang A-G, et al. G*Power 3: a flexible statistical power analysis program for the social, behavioral, and biomedical sciences. Behav Res Methods 2007;39:175-91.

48 Vancampfort D, Rosenbaum S, Schuch FB, et al. Prevalence and predictors of treatment dropout from physical activity interventions in schizophrenia: a meta-analysis. Gen Hosp Psychiatry 2016;39:15-23.

49 Noordsy DL, Burgess JD, Hardy KV, et al. Therapeutic potential of physical exercise in early psychosis. AJP 2018;175:209-14.

50 Sabe M, Kaiser S, Sentissi O. Physical exercise for negative symptoms of schizophrenia: systematic review of randomized controlled trials and meta-analysis. Gen Hosp Psychiatry 2020;62:13-20.

51 Pearsall R, Smith DJ, Pelosi A, et al. Exercise therapy in adults with serious mental illness: a systematic review and meta-analysis. BMC Psychiatry 2014;14:117.

52 Borg GA. Psychophysical bases of perceived exertion. Med Sci Sports Exerc 1982;14:377???381-81.

53 Tanaka H, Monahan KD, Seals DR. Age-predicted maximal heart rate revisited. J Am Coll Cardiol 2001;37:153-6.

54 Kay SR, Fiszbein A, Opler LA. The positive and negative syndrome scale (PANSS) for schizophrenia. Schizophr Bull 1987;13:261-76.

55 Kay SR, Fiszbein A, Vital-Herne M, et al. The Positive and Negative Syndrome Scale--Spanish adaptation. J Nerv Ment Dis 1990;178:510-7.

56 Booth M. Assessment of physical activity: an international perspective. Res Q Exerc Sport 2000;71:114-20.

57 The IPAQ group. International physical activity questionnaire, 2021 Available: https://sites.google.com/site/theipaq/ [Accessed 12 Mar 2021].

58 Craig CL, Marshall AL, Sjöström M, et al. International physical activity questionnaire: 12-country reliability and validity. Med Sci Sports Exerc 2003;35:1381-95

59 Román BV, Ribas LB, Ngo J. [Validity of the international physical activity questionnaire in the Catalan population (Spain)]. Gac Sanit 2013;27.

60 WHOQOL Group. Study protocol for the world Health organization project to develop a quality of life assessment instrument (WHOQOL). Qual Life Res 1993;2:153-9.

61 Espinoza I, Osorio P, Torrejón MJ, et al. [Validation of the WHOQOLBREF quality of life questionnaire among Chilean older people]. Rev Med Chil 2011;139:579-86.

62 Cohen J. Statistical power analysis for the behavioral sciences. 2 edn. United States of America: Lawrence Erlbaum Associates, 1988.

63 Pajonk F-G, Wobrock T, Gruber O, et al. Hippocampal plasticity in response to exercise in schizophrenia. Arch Gen Psychiatry 2010;67:133-43

64 Cheng S-L, Sun H-F, Yeh M-L. Effects of an 8-Week aerobic dance program on health-related fitness in patients with schizophrenia. $J$ Nurs Res 2017;25:429-35.

65 Ren J, Xia J. Dance therapy for schizophrenia. Cochrane Database Syst Rev 2013:CD006868.

66 Cassilhas RC, Antunes HKM, Tufik S, et al. Mood, anxiety, and serum IGF-1 in elderly men given 24 weeks of high resistance exercise. Percept Mot Skills 2010;110:265-76.

67 Stanton R, Reaburn P, Happell B. Is cardiovascular or resistance exercise better to treat patients with depression? A narrative review. Issues Ment Health Nurs 2013;34:531-8.

68 Hidese S, Matsuo J, Ishida I, et al. Relationship of handgrip strength and body mass index with cognitive function in patients with schizophrenia. Front Psychiatry 2018;9:156.

69 Firth JA, Smith L, Sarris J, et al. Handgrip strength is associated with hippocampal volume and white matter hyperintensities in major depression and healthy controls: a UK Biobank study. Psychosom Med 2020;82:39-46.

70 Subramaniapillai M, Arbour-Nicitopoulos K, Duncan M, et al. Physical activity preferences of individuals diagnosed with schizophrenia or bipolar disorder. BMC Res Notes 2016;9:340.

71 Noordsy DL, Burgess JD, Hardy KV, et al. Therapeutic potential of physical exercise in early psychosis. Am J Psychiatry 2018;175:209-14.

72 Firth J, Cotter J, Carney R, et al. The pro-cognitive mechanisms of physical exercise in people with schizophrenia. Br J Pharmacol 2017;174:3161-72. 